\title{
Analysis of Linear Antenna Array for minimum Side Lobe Level, Half Power Beamwidth, and Nulls control using PSO
}

\author{
Saeed Ur Rahman ${ }^{1}$, Qunsheng CAO ${ }^{1}$, \\ ${ }^{1}$ College of Electronics and Information Engineering, \\ Nanjing University of Aeronautics and Astronautics (NUAA), \\ Nanjing 2111016, China \\ saeed@nuaa.edu.cn , qunsheng@nuaa.edu.cn \\ Muhammad Mansoor Ahmed², Hisham Khali1 ${ }^{2}$ \\ ${ }^{2}$ Department of Electrical Engineering, \\ Capital University of Science and Technology (CUST), Islamabad, Pakistan. \\ mansoor@cust.edu.pk, hisham.hamidi@gmail.com
}

\begin{abstract}
This paper presents the optimization performance of non-uniform linear antenna array with optimized inter-element spacing and excitation amplitude using Particle Swarm Optimization (PSO). The aim of the proposed algorithm is to obtain the optimum values for inter-element spacing and excitation amplitude for a linear antenna array in a given radiation pattern with suppressed Side Lobe Level (SLL), minimum Half Power Beamwidth (HPBW), improved directivity and placement of nulls in the desired direction. A variety of design examples are considered and the obtained results using PSO are validated by benchmarking with results obtained using other nature-inspired meta-heuristic algorithms such as the Real-coded Genetic Algorithm (RGA) and the Biogeographic Based Optimization (BBO) algorithm. The comparative results are shown that optimization of linear antenna array using the PSO provides considerable enhancement in the SLL, the HPBW, the directivity and the null control in the desired direction.
\end{abstract}

Index Terms - Linear Antenna Array, Improved Directivity, Null Control, Particle Swarm Optimization, Minimum Side Lobe Level, Half Power Beamwidth.

\section{INTRODUCTION}

An antenna array is a combination of two or more antenna elements that can be placed in a specific geometry. In a linear antenna array, antenna elements are placed along one axis. The antenna array produces a beam, this beam can effect by changing the geometry (linear, circular, spherical etc.) and also by some other parameters i.e. inter-element spacing, excitation amplitude and excitation phase of the individual element [1]. In mostly wireless communication requires more directive antenna having high gain. Antenna array has high gain, more directive, spatial diversity. Antenna array synthesis has received importance in the near past, where various performance goals were considered. For array synthesis, different optimization algorithms e.g., the simulated annealing [2], the ant-colony 
optimization [3], the GA [4] and the PSO [5] have been used in the previous research studies. The optimization of linear antenna provides a pattern that has minimum the SLL and the HPBW i.e. by using the PSO and the GA [5]-[7]. The composite differential evaluation (CoDE) algorithm applied to optimize inter-element spacing, between two consecutive elements to minimize the SLL and to place nulls in the desired direction [8-9]. A multi-objective optimization approach has been used in Ref. [10]-[11], to maximize the directivity and to minimize the SLL of an antenna array in the optimization process. In Ref. [10] the authors introduced a new technique memetic multi-objective evolutionary algorithm called memetic generalized differential evaluation (MGDE3) algorithm, which is the extension of generalized differential evaluation (GDE3) algorithm. In Ref. [12] the authors introduced a technique to realize the characteristics and performance of the non-uniform linear array. The presented technique had been utilized multi-objective functions to optimize inter-elements spacing, excitation currents, and excitation phases as well as minimized SLL and HPBW. Real-coded genetic (RCG) was employed for time modulating linear antenna array to impose nulls in the desired direction by optimizing spacing and excitation amplitude.

In this paper, two fitness function are presented. One fitness function is defined to place nulls in the desired direction by optimizing excitation amplitude, another one is provided a pattern of minimized SLL and HPBW. The paper is arranged as follow. Section II is addressed the analysis of design parameters, the number of the element, inter-element spacing, and excitation amplitude, of a linear antenna array. In section III, the PSO and optimizing the design parameters using the PSO are briefly described and studied in section IV and section V, respectively. The PSO employed to improve directivity, placing nulls in the desired direction are discussed in section in V. Finally, Section VI states the conclusions.

\section{ANALYSIS OF ARRAY FACTOR}

The linear antenna array parameters generally involve inter-element spacing, excitation amplitude, and number of elements, which directly influences its effect on array factor (AF). All these variables have been used to demonstrate beam forming and beam steering of a linear array. Fig. 1 shows a linear antenna array in which identical antenna elements are placed on one side from the origin.

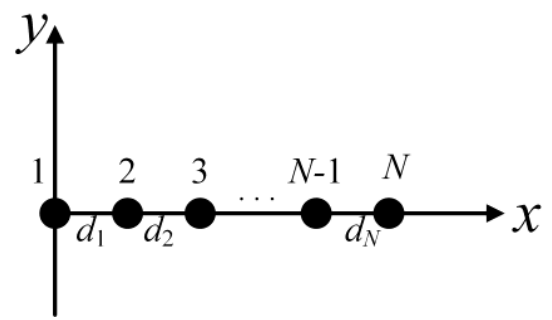

Fig. 1. The geometry of $\mathrm{N}$ elements along the $\mathrm{x}$-axis.

The AF for $N$ elements can be considered as follows[13]. 


$$
A F=\sum_{i=1}^{N} a_{i} e^{\left(k d_{i} \cos \theta+\phi_{i}\right)}
$$

where $N$ is the number of antenna elements, $a_{i}, d_{i}, \phi_{i}$, and $k$ are the excitation amplitude, the inter-element spacing, the excitation phase and the propagation constant for the $i^{\text {th }}$ element. To study the effects of these variables for an optimum design, a MATLAB routine has been developed using Eq. (1), and the $\mathrm{AF}$ has been plotted as a function of various control parameters.

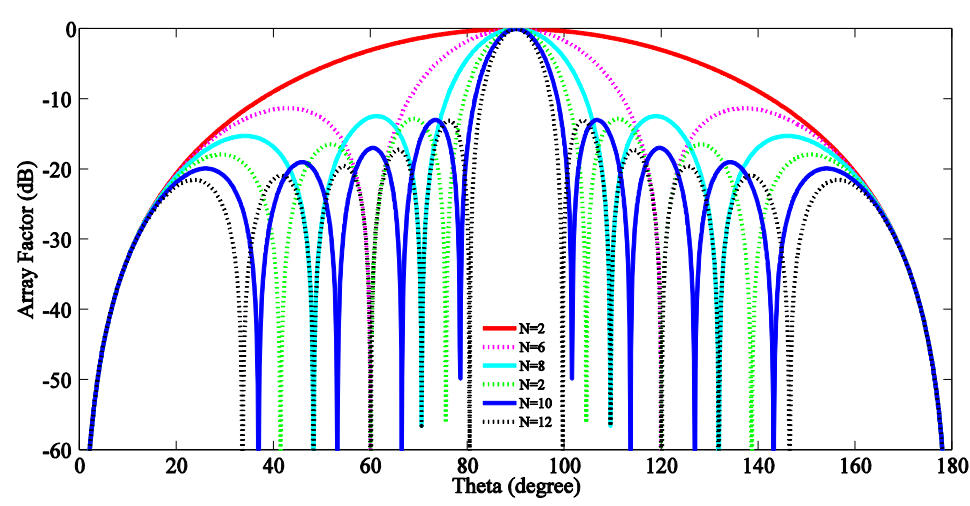

Fig. 2. AF varied for element $\mathrm{N}$ from 2 to 12

Because the element $N$ in an antenna array plays an important role in beam forming, beam steering, and interference reduction. Fig. 2 is plotted by varying the number of elements while keeping the spacing between two consecutive elements as $0.5 \lambda$ and $\phi_{i}=90^{\circ}$. Results of Fig. 2 are normalized with respect to the maximum value of the main lobe. From the Fig. 2, it has found that the HPBW is decreased with a minor reduction in the SLL with an increase in the number of elements.

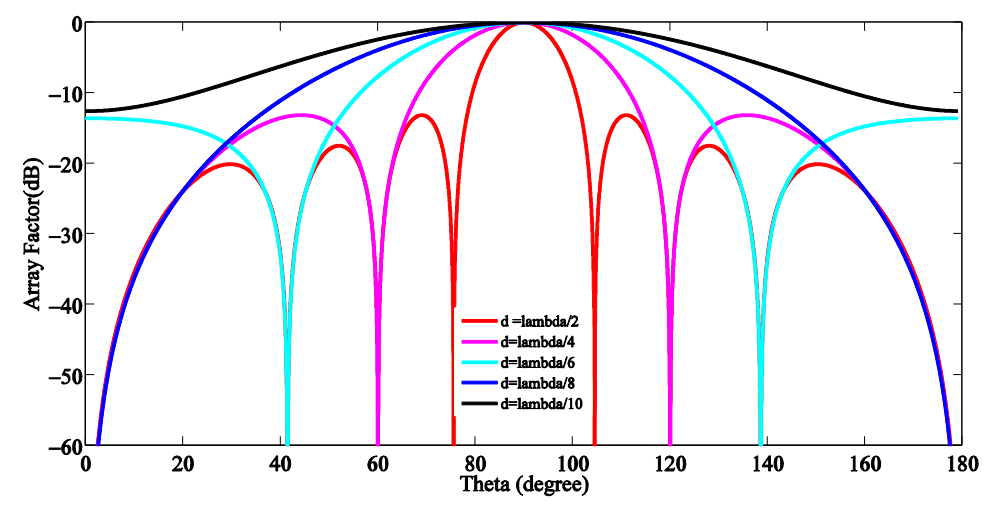

Fig. 3. AF varied with distance, $d$ decreasing from $\lambda / 2$ to $\lambda / 10$.

The performance of an antenna array is also dependent on the distance between two consecutive elements. The distance between the elements should be close to $0.5 \lambda$. To observe the effect of spacing, the distance $d$ on the radiation characteristics of an antenna array, a simulation has been carried out with $d$ changing from $\lambda / 2$ to a relatively small Brazilian Microwave and Optoelectronics Society-SBMO received 23 Feb 2017; for review 02 Mar 2017; accepted 14 May 2017 Brazilian Society of Electromagnetism-SBMag 
separation between two array elements, i.e., up to $d=\lambda / 10$ shown in Fig. 3. It has demonstrated that the distance $d$ should be closer to $\lambda / 2$.

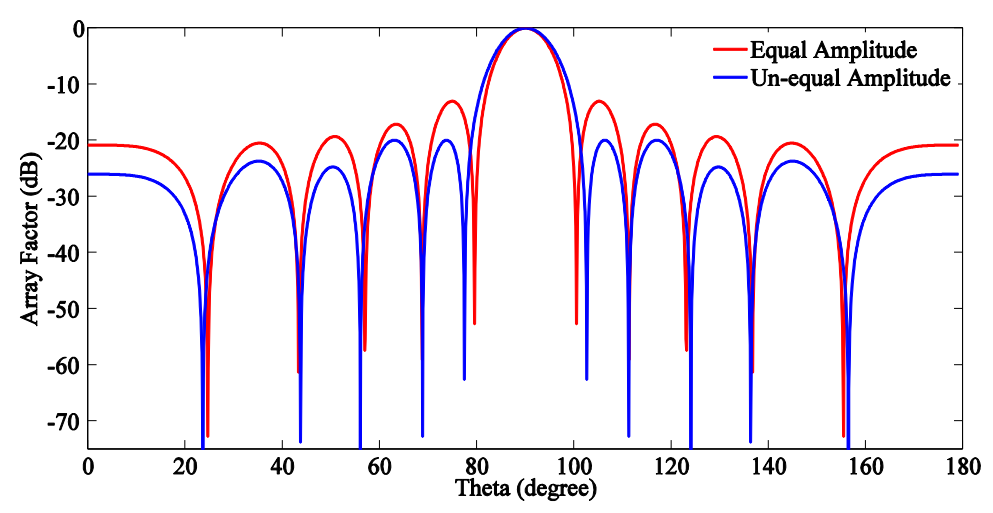

Fig. 4. AF varied for equal and unequal excitation amplitude.

Excitation amplitude for each individual element, commonly known as a weight factor, also changes radiation characteristics of an array antenna. By changing its value for various elements of an array, one can change the overall array pattern. A comparative analysis of weighted and un-weighted antenna design is presented in Fig. 4. This is a primitive analysis, which is not based on the appropriately weighted amplitudes rather than the amplitudes are changed to observe its effect on AF variation. For equal and unequal amplitude excitation, the HPBW is decreased and the SLL is increased while for unequal amplitude excitation, the SLL is reduced and the HPBW is increased shown in Fig. 4.

\section{PARTICLE SWARM OPTIMIZATION ALGORITHM}

The PSO is frequently used to solve successfully complex multidimensional optimization problems in different fields, such as antenna design and devicemodeling [14]-[25] etc. In the PSO, every individual entity in the swarm is referred to as a particle and is associated with a velocity. All particles move in search of space and update their velocity according to the best position which is already found by themselves and by their neighbor which is also trying to find the best position. The PSO is a computational method which iteratively optimizes the problem based on a certain fitness function. Since we are dealing with both uniform and non-uniform linear antenna array, so each particle is a vector which contains the information for each feed antenna. This information includes the excitation amplitude $\left|a_{i}\right|$, excitation phase $\left|\phi_{i}\right|$ and the distance of element with respect to the previous position, $d$ in the array. The flow chart of the PSO is given in Fig. 5 and the PSO algorithm can be summarized as:

For each particle $i=1 \ldots S$.

i. Generate random $d_{i}, a_{i}$ and $\phi_{\mathrm{i}}$ where $\mathrm{x}_{\mathrm{i}}=\left[\mathrm{d}_{\mathrm{i}}\left|\mathrm{a}_{\mathrm{i}}\right| \phi_{\mathrm{i}}\right]$ and range for $|a|,|\phi|$ and $d$ is $[0,1]$, $\left[0,360^{0}\right],\left[d_{\max }, d_{\min }\right]$, respectively.

ii. Initialize individual particle velocities:

$$
\begin{aligned}
& v_{i, d}=U\left(-\left|d_{\text {max }}-d_{\text {min }}\right|,\left|d_{\text {max }}-d_{\text {min }}\right|\right) \\
& v_{i,|a|}=U\left(-|a|_{\text {max }}-|a|_{\text {min }}|,||a|_{\text {max }}-|a|_{\text {min }} \mid\right)
\end{aligned}
$$


$v_{i, \phi}=U\left(-\left|\phi_{\max }-\phi_{\min }\right|,\left|\phi_{\max }-\phi_{\min }\right|\right)$

iii. Compute fitness function for each particle.

Compute the particle's best position $\left(\mathrm{p}_{\mathrm{i}}\right)$ and the global best position $(\mathrm{g})$ such that $\mathrm{f}(\mathrm{pi}$ ) $<\mathrm{f}(\mathrm{g})$

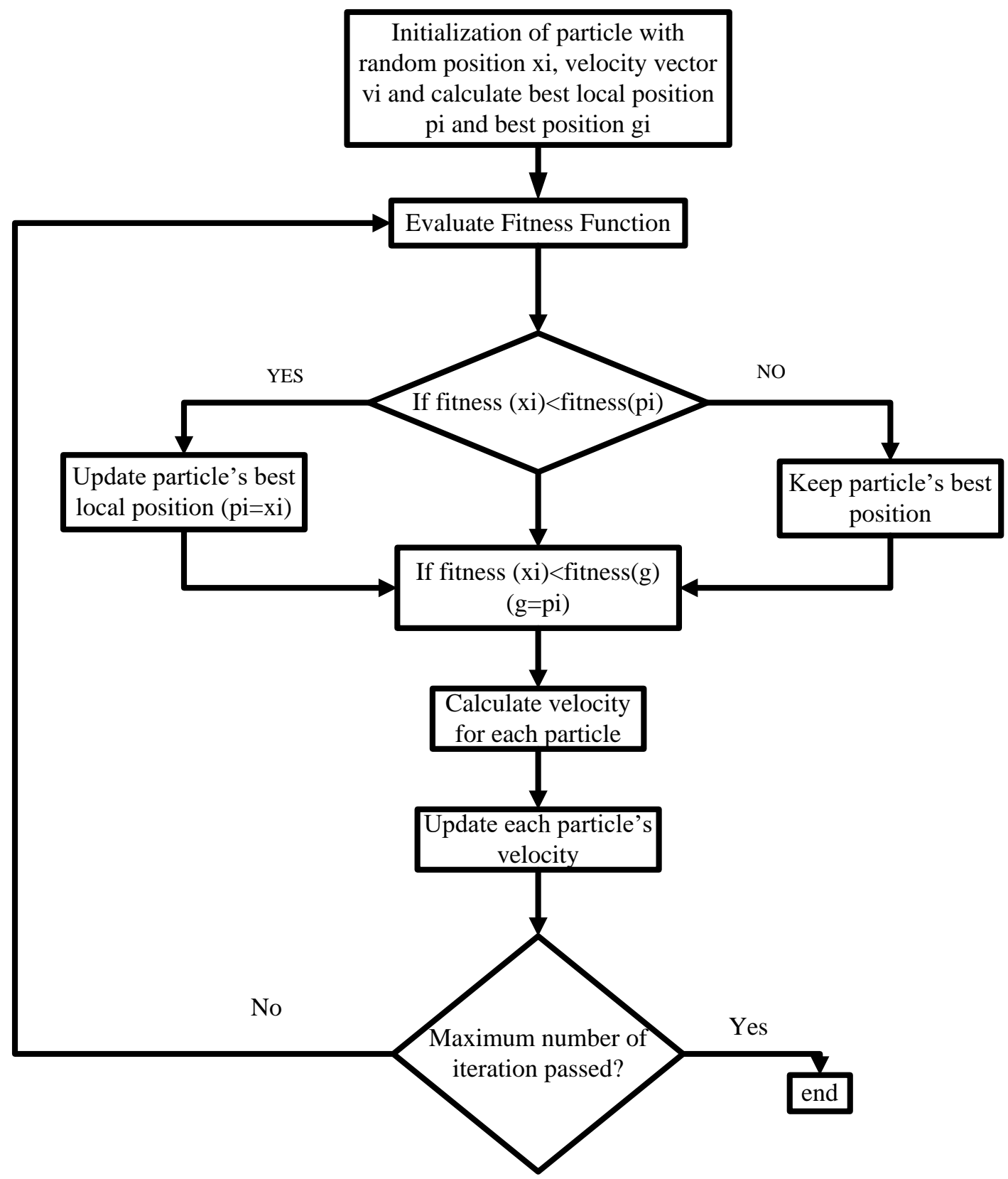

Fig. 5. Flow chart of PSO

After the initialization phase, the optimization steps are as follows:

i. $\quad$ For each particle $i=1, \ldots, \mathrm{S}$ do:

ii. Pick uniformly distributed random numbers for the best local $r_{p}$ and global position $r_{g}$.

iii. Update the velocity for each dimension: 


$$
\begin{aligned}
& v_{i, d}=\beta v_{i, d}+\alpha_{p} r_{p}\left(p_{i, d}-x_{i, d}\right)+\alpha_{g} r_{g}\left(g_{d}-x_{i, d}\right) \\
& v_{i,|a|}=\beta v_{i,|a|}+\alpha_{p} r_{p}\left(p_{i,|a|}-x_{i,|a|}\right)+\alpha_{g} r_{g}\left(g_{a}-x_{i,|a|}\right) \\
& v_{i, \angle a}=\beta v_{i, \angle a}+\alpha_{p} r_{p}\left(p_{i, \angle a}-x_{i, \angle a}\right)+\alpha_{g} r_{g}\left(g_{\angle a}-x_{i, \angle a}\right)
\end{aligned}
$$

where $\beta=1, \alpha_{p}=0.9, \alpha_{\mathrm{g}}=0.9$

After updating velocity this algorithm will repeat till certain termination criteria like a total number of iteration are met. The PSO requires appropriate fitness function for the algorithm. A fitness function should have the ability to reduce the SLL and minimize the HPBW. A fitness function is designed considering the target value of the SLL and the HPBW. It is given in (2) and (3).

\section{SYNTHESIS OF LINEAR ANTENNA ARRAY USING PSO}

In this section, the PSO is employed for optimum values of excitation amplitude and inter-element spacing to minimize the SLL and HPBW. In order to compare with previous research work, three elements $10,12,16$ are considered as examples to simulate.

$$
\begin{gathered}
f_{1}=\left\{\begin{array}{l}
\min (H P B W)-\min (S L L) \quad \text { if } \min (S L L) \leq A_{0} \& \min (H P B W) \leq B_{0} \\
\left.\min (H P B W)-A_{0}\right)-\left(\max (S L L)-B_{0}\right)
\end{array}\right. \\
f_{2}=\max \left(A F_{1}\left(\theta_{d}\right)\right)
\end{gathered}
$$

where $A F_{1}=|A F|^{2}, \mathrm{~A}_{0}$ and $\mathrm{B}_{0}$ are the fixed values of the HPBW and the SLL that keep the SLL and the HPBW less than $\mathrm{A}_{0}$ and $\mathrm{B}_{0}$, and $\theta_{\mathrm{d}}$ is the angle at which null can be controlled. In the first section fitness function ' $f_{l}$ ' is employed to minimize the SLL so as to get the optimum values of excitation amplitude and inter-element spacing. While in the second section ' $f_{2}$ ' is used to control the nulls in a specified direction.

\section{A. Optimization of Excitation Amplitude}

For each individual element, the excitation amplitude, also known as the weighting factor, are changed the characteristics of the antenna array. 10, 12, and 16 antenna elements arrays are considered to optimize the excitation amplitude in order to achieve the suppressed SLL and the minimum HPBW. The inter-element spacing and excitation phase are fixed i.e., the inter-element spacing between two adjacent antenna elements is $0.5 \lambda$ while excitation phase for each element is $90^{\circ}$. 


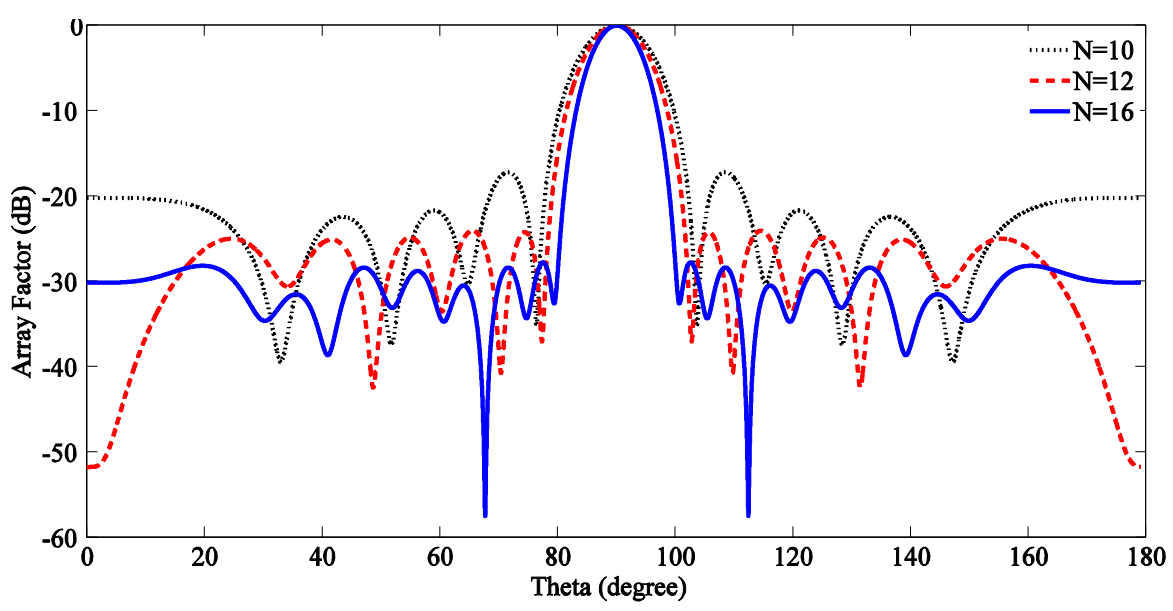

Fig. 6. AF varied with optimized excitation amplitude

TABLE.I. COMPARATIVE RESULTS FOR OPTIMIZED EXCITATION AMPLITUDE.

\begin{tabular}{|c|c|c|c|c|c|c|}
\hline & \multicolumn{3}{|l|}{ Proposed PSO } & BBO [26] & CFO [27] & GA [28] \\
\hline No. elements & 10 & 12 & 16 & 10 & 10 & 16 \\
\hline Max SLL (dB) & -17.26 & -24.14 & -28.05 & -15.97 & -15.93 & -16.07 \\
\hline HPBW(degree) & 11 & 9 & 7 & - & - & - \\
\hline
\end{tabular}

TABLE. II. OPTIMIZED VALUES FOR EXCITATION AMPLITUDE WITH FIXED SPACING AND PHASE

\begin{tabular}{|c|l|l|l|}
\hline amplitude & $\mathrm{N}=16$ & $\mathrm{~N}=12$ & $\mathrm{~N}=10$ \\
\hline $\mathrm{a}_{1}$ & 0.2127 & 0.3087 & 0.3881 \\
\hline $\mathrm{a}_{2}$ & 0.2287 & 0.3938 & 0.4097 \\
\hline $\mathrm{a}_{3}$ & 0.3606 & 0.4444 & 0.6289 \\
\hline $\mathrm{a}_{4}$ & 0.3606 & 0.5530 & 0.6322 \\
\hline $\mathrm{a}_{5}$ & 0.5198 & 0.7159 & 0.6778 \\
\hline $\mathrm{a}_{6}$ & 0.5627 & 0.7115 & 0.6008 \\
\hline $\mathrm{a}_{7}$ & 0.6373 & 0.7725 & 0.6012 \\
\hline $\mathrm{a}_{8}$ & 0.6496 & 0.6733 & 0.4768 \\
\hline $\mathrm{a}_{9}$ & 0.7127 & 0.6166 & 0.5620 \\
\hline $\mathrm{a}_{10}$ & 0.7088 & 0.5146 & 0.2291 \\
\hline $\mathrm{a}_{11}$ & 0.5694 & 0.3158 & \\
\hline $\mathrm{a}_{12}$ & 0.5113 & 0.3113 & \\
\hline $\mathrm{a}_{13}$ & 0.4545 & & \\
\hline $\mathrm{a}_{14}$ & 0.2761 & & \\
\hline $\mathrm{a}_{15}$ & 0.2228 & & \\
\hline $\mathrm{a}_{16}$ & 0.1693 & & \\
\hline & & & \\
\hline
\end{tabular}


The excitation amplitude is assumed as the range [0 1]. For uniform inter-element spacing and excitation phase, the proposed PSO algorithm has a peak value of $-17.26 \mathrm{~dB}$ with the minimum HPBW as $11^{\circ}$ when 10 antenna elements are selected. For $\mathrm{N}=12$ case, the peak SLL is less than $-24.14 \mathrm{~dB}$ while the HPBW decreases to $9^{\circ}$. Similarly, for $\mathrm{N}=16$ case the peak SLL is further reduced to $-28.05 \mathrm{~dB}$ and the HPBW is less than $7^{\circ}$ shown in Fig. 6. It has been found that there is an improvement of $1.29 \mathrm{~dB}$ and $11.98 \mathrm{~dB}$ in the SLL compared with the results of the $\mathrm{BBO}$ [26] and the GA [28] for $\mathrm{N}=10$ and $\mathrm{N}=16$, respectively. Considerable improvement in the HPBW has been observed while the SLL is suppressed with increasing number of elements listed in Table I.

\section{B. Optimization of Inter-element spacing}

The performance of an antenna array is also dependent on the distance between two consecutive elements. To obtain the optimum values of inter-element spacing, the PSO algorithm has been employed with equal excitation amplitude $a_{\mathrm{i}}=1$ and excitation phase $\phi_{\mathrm{i}}=$ $90^{\circ}$ by using the fitness function $\mathrm{f}$ associated with the SLL and HPBW. The number of elements in the antenna array has been chosen as 10,12 , and 16 .

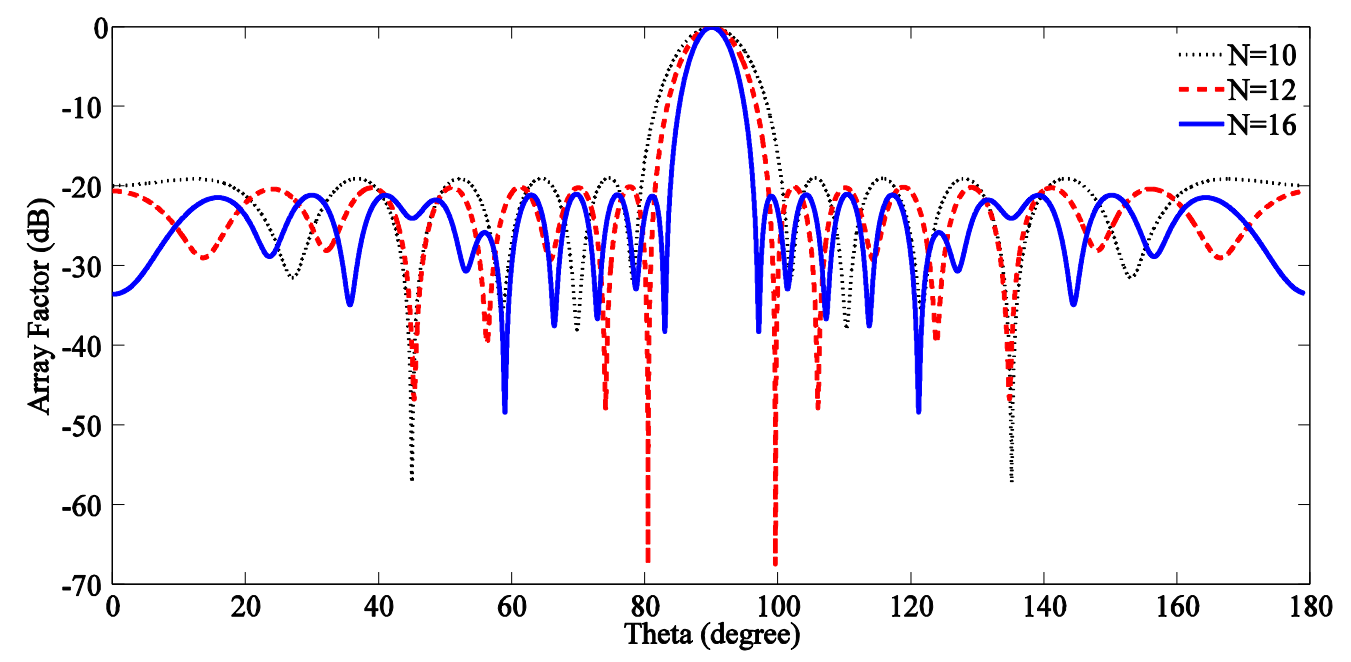

Fig. 7. Plot of AF when inter-element spacing was optimized

TABLE.III. COMPARATIVE RESULTS FOR OPTIMIZED INTER-ELEMENT SPACING.

\begin{tabular}{|c|c|c|c|c|c|}
\hline & \multicolumn{3}{|c|}{ Proposed PSO } & BBO [26] & PSO [29] \\
\hline No. of elements & 10 & 12 & 16 & 10 & 10 \\
\hline Max SLL (dB) & -19.10 & -20.09 & -21.08 & -18.14 & -17.82 \\
\hline HPBW(degree) & 11 & 7 & 5 & - & 8.9 \\
\hline
\end{tabular}

The optimized values of inter-element spacing are given in Table IV and comparative results are given in Table III. It has been observed that the HPBW is minimized up to $5^{\circ}$ and the SLL is reduced up to $-21.08 \mathrm{~dB}$ when $\mathrm{N}$ was increased up to 16 . As compared with the results of the BBO [26] and the PSO [29] there is an improvement of $1.0 \mathrm{~dB}$ in the SLL and $2.1^{\circ}$ in the HPBW by using the proposed algorithm shown in Fig. 7 
TABLE. IV. OPTIMIZED VALUES FOR SPACING WITH FIXED EXCITATION AMPLITUDE AND PHASE.

\begin{tabular}{|c|c|c|c|}
\hline Spacing & $\mathrm{N}=16$ & $\mathrm{~N}=12$ & $\mathrm{~N}=10$ \\
\hline $\mathrm{d}_{1}$ & 0.7851 & 0.7141 & 0.7022 \\
\hline $\mathrm{d}_{2}$ & 0.8143 & 0.7070 & 0.5621 \\
\hline $\mathrm{d}_{3}$ & 0.6368 & 0.5496 & 0.4684 \\
\hline $\mathrm{d}_{4}$ & 0.5386 & 0.5183 & 0.4233 \\
\hline $\mathrm{d}_{5}$ & 0.5820 & 0.4100 & 0.4780 \\
\hline $\mathrm{d}_{6}$ & 0.4491 & 0.4719 & 0.4932 \\
\hline $\mathrm{d}_{7}$ & 0.5882 & 0.4884 & 0.6535 \\
\hline $\mathrm{d}_{8}$ & 0.4074 & 0.5118 & 0.6926 \\
\hline $\mathrm{d}_{9}$ & 0.4277 & 0.6436 & 0.4929 \\
\hline $\mathrm{d}_{\mathrm{d} 0}$ & 0.4955 & 0.8274 & \\
\hline $\mathrm{d}_{11}$ & 0.5096 & 0.5958 & \\
\hline $\mathrm{d}_{12}$ & 0.4741 & & \\
\hline $\mathrm{d}_{13}$ & 0.7078 & & \\
\hline $\mathrm{d}_{14}$ & 0.7736 & & \\
\hline $\mathrm{d}_{15}$ & 0.8566 & & \\
\hline & & & \\
\hline
\end{tabular}

\section{Optimization of Excitation Amplitude and Inter-element spacing}

The PSO method is also employed to optimize the excitation amplitude and inter-element spacing. The fitness function given by Eq. (2) is used to get the optimum amplitude and position so as to minimize the SLL and the HPBW. In Table V, the observations are made for $\mathrm{N}=12,16$ and the results are compared with previous research work [1].

TABLE. V. COMPARATIVE RESULTS FOR MINIMUM SLL AND HPBW WHEN N $=12,16$

\begin{tabular}{|c|c|c|c|c|}
\hline & \multicolumn{2}{|c|}{ Proposed PSO } & \multicolumn{2}{c|}{ RGA[1] } \\
\hline No. of elements & 12 & 16 & 12 & 16 \\
\hline Max SLL (dB) & -28.29 & -31.29 & -13.56 & -15.18 \\
\hline HPBW(degree) & 9 & 7 & 9.55 & 8.45 \\
\hline
\end{tabular}

As compared to [1] there is an improvement of $13.85 \mathrm{~dB}$ in the SLL and $0.45^{\circ}$ in the HPBW by using the PSO and similarly, for $\mathrm{N}=16$, there is an improvement of $16.11 \mathrm{~dB}$ in the SLL and $0.45^{\circ}$ in the HPBW shown in Fig. 8 . 


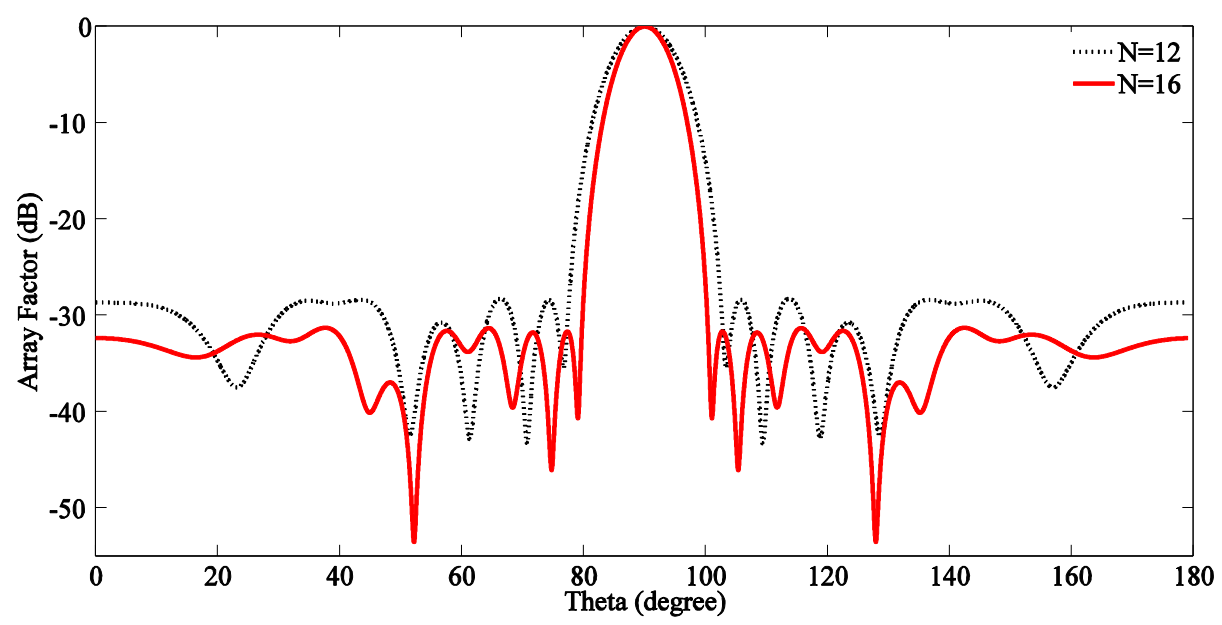

Fig. 8. AF varied with optimized inter-element spacing and excitation amplitude

\section{DIRECTIVITY AND NULL CONTROL}

The directivity of the antenna can be defined as the ratio of radiation intensity in a given direction from the antenna /antenna array to the radiation intensity averaged over all directions [13]. The mathematical form of directivity, D for antenna arrays can be written as

$$
\begin{gathered}
D=\frac{4 \pi U_{\text {max }}}{P_{\text {rad }}} \\
U_{\text {max }}=\frac{|A F|}{\max (|A F|)^{2}} \\
P_{\text {rad }}=\int_{0}^{2 \pi \pi} \int_{0}^{2 \pi} U(\theta) \sin (\theta) d \theta d \phi
\end{gathered}
$$

where $\mathrm{P}_{\mathrm{rad}}$ is the total radiated power and $\mathrm{U}_{\max }$ is the maximum radiation intensity.

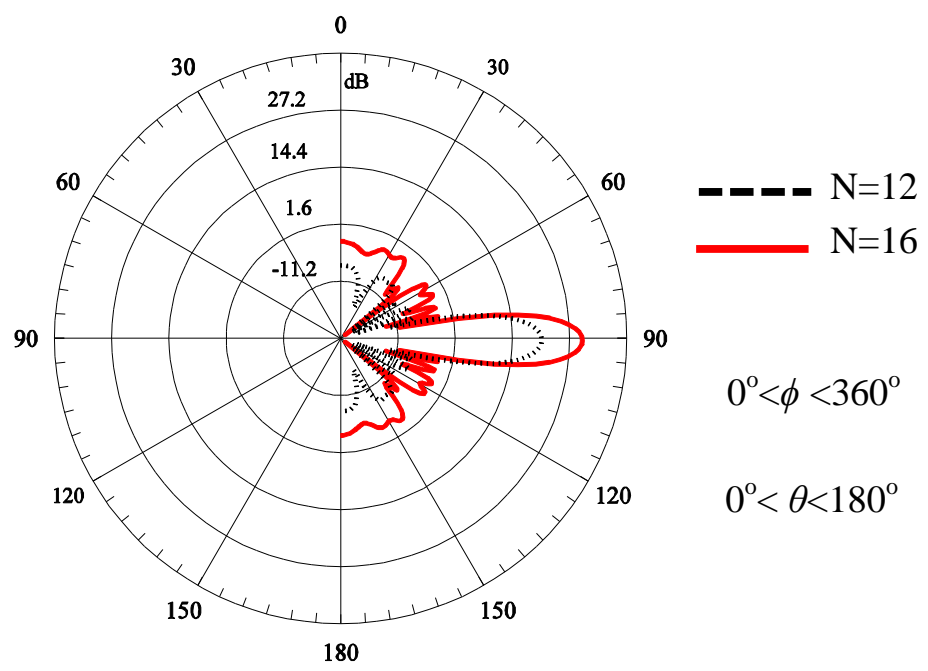

Fig. 9. 2D plot of directivity for optimized excitation amplitude and inter-element spacing 
The directivity of the linear antenna array can be improved by controlling the switching time [30], inter-element spacing and excitation amplitude. Considering 12 and 16 antenna array elements, the directivity and the SLL of linear antenna arrays have been improved by increasing the number of elements and controlling the inter-element spacing, the excitation amplitude. table VI. is listed the comparative results for the directivity and the SLL.

TABLE VI. COMPARATIVE RESULTS FOR DIRECTIVITY AND SLL
\begin{tabular}{|c|l|l|l|}
\hline No. of elements & Algorithms & Directivity (dB) & SLL (dB) \\
\hline 12 & Proposed & 10.4100 & -28.29 \\
\hline 16 & Proposed & 11.3764 & -31.29 \\
\hline 16 & DE[30] & 11.4683 & -18.74 \\
\hline 16 & PSO[30] & 11.4515 & -18.73 \\
\hline 16 & RGA[30] & 11.4027 & -17.8 \\
\hline
\end{tabular}

Another important application of linear array is null control [1], [5]-[6], [9]. The null control refers to control the radiation pattern in a way such that a relatively small amount of power is received/radiated in certain directions. At the transmitting end, the null control is used for transmitting low power in the directions where an eavesdropper is present. On the receiving side, it is used to reduce the amount of power received from interferers. The null control can be achieved by controlling the parameters, excitation amplitude, excitation phase, array spacing and the number of elements. It is important to note that reducing the amount of power in one direction means that power is increased in another direction. Ideally, the power is decreased in the direction of interferers and the main beam is increased in the same direction. Generally, it is hard to accomplish this, it is needed to tradeoff. So the PSO algorithm is employed to place nulls in specified directions using the fitness function $f_{2}$. Three different examples are considered for different antenna elements and nulls at specified places.

TABLE VII. SLL, HPBW AND NULL DEPTH WHEN NULLS ARE IMPOSED AT $\theta_{d}=50^{\circ}, 55^{\circ}, 125^{\circ}$ AND $130^{\circ}$
\begin{tabular}{|c|c|c|c|c|}
\hline N & HPBW (degree) & SLL (dB) & Null depth at $50^{\circ}$ & Null depth at $55^{\circ}$ \\
\hline 10 & 11 & -10 & $-55.9 \mathrm{~dB}$ & $-55.5 \mathrm{~dB}$ \\
\hline 12 & 9 & -11 & $-48.1 \mathrm{~dB}$ & $-49.1 \mathrm{~dB}$ \\
\hline 16 & 7 & -11.6 & $-57 \mathrm{~dB}$ & $-57 \mathrm{~dB}$ \\
\hline
\end{tabular}

In order to validate the PSO algorithm, a linear antenna array of $\mathrm{N}=10,12$ and 16 elements is analyzed, respectively, with the nulls imposed at $\theta=50^{\circ}, 55^{\circ}, 125^{\circ}$ and $130^{\circ}$. The PSO is employed with the appropriate fitness function to control the nulls at specified angular direction. Table VII is listed the values of the SLL, the HPBW and the null depth for imposed nulls at $\theta=50^{\circ}, 55^{\circ}, 125^{\circ}$ and $130^{\circ}$. 


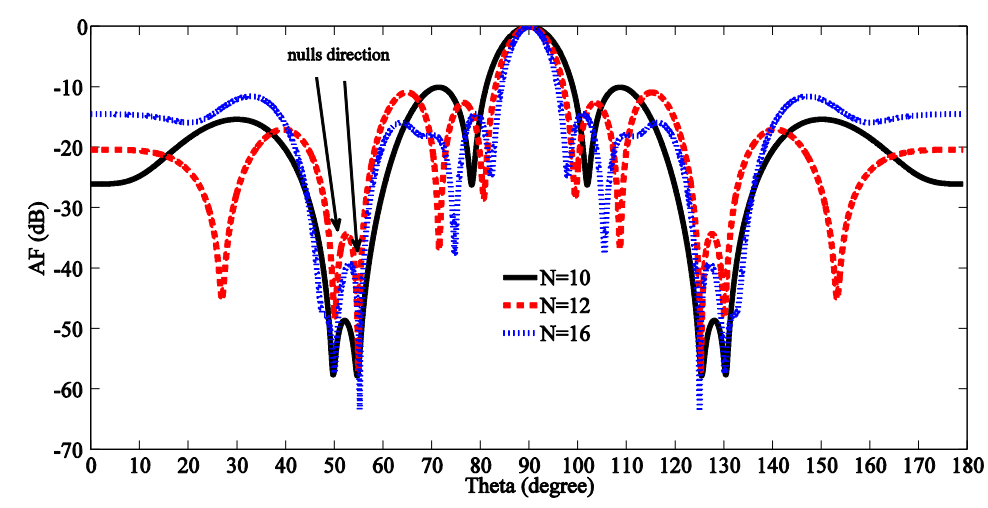

Fig. 10. Plot of AF when nulls are imposed at $\theta=50^{\circ}, 55^{\circ}, 125^{\circ}$ and $130^{\circ}$.

TABLE VIII. OPTIMIZED VALUES OF EXCITATION AMPLITUDE WHEN NULLS ARE PLACED AT $\theta_{d}=50^{0}, 55^{\circ}, 125^{\circ}$ AND

\begin{tabular}{|c|c|c|c|}
\hline No. of elements & 10 & 12 & 16 \\
\hline \multirow{16}{*}{ Excitation Amplitude } & 0.2658 & 0.3465 & 0.3931 \\
\hline & 0.3578 & 0.7744 & 0.2958 \\
\hline & 0.8053 & 0.5334 & 0.5136 \\
\hline & 0.4794 & 0.5146 & 0.5263 \\
\hline & 0.4630 & 0.2835 & 0.4735 \\
\hline & 0.2575 & 0.6050 & 0.7563 \\
\hline & 0.3758 & 0.7625 & 0.1670 \\
\hline & 0.6736 & 0.3483 & 0.5305 \\
\hline & 0.4199 & 0.3025 & 0.7270 \\
\hline & 0.3397 & 0.5249 & 0.5964 \\
\hline & & 0.5333 & 0.9566 \\
\hline & & 0.5828 & 0.0093 \\
\hline & & & 0.8880 \\
\hline & & & 0.4377 \\
\hline & & & 0.7248 \\
\hline & & & 0.1519 \\
\hline
\end{tabular}

Fig. 10 is plotted the AF is varied for imposed nulls at $\theta=50^{\circ}, 55^{\circ}, 125^{\circ}$ and $130^{\circ}$. Table VIII is given the optimized value of inter-element spacing for the nulls located at $\theta=50^{\circ}, 55^{\circ}, 125^{\circ}$ and $130^{\circ}$.

Next, in order to study deep null at the first nulls of the main beam, the PSO algorithm is employed using fitness function $f_{2}$ as given in Eq. (3). The first nulls for the main beam are placed at $80^{\circ}$ and $100^{\circ}$. The purpose of these nulls is to reject the interfering signal close to the main beam and also to minimize the HPBW. The null depth is around $-54 \mathrm{~dB}$ at $80^{\circ}$ and $100^{\circ}$ while it is around $-59 \mathrm{~dB}$ at $70^{\circ}$ and $110^{\circ}$ where minimum SLL is $-16 \mathrm{~dB}$ for $\mathrm{N}=16$. Table $X$ is listed the SLL, the HPBW and the nulls depth imposed at $\theta=70^{\circ}, 80^{\circ}, 100^{\circ}$ and $130^{\circ}$ for $\mathrm{N}=10,1216$. 
TABLE IX. OPTIMIZED VALUES OF EXCITATION AMPLITUDES WHEN NULLS ARE IMPOSED AT $\theta=70^{\circ}, 80^{\circ}, 100^{\circ}$ AND

\begin{tabular}{|c|c|c|c|}
\hline \multicolumn{4}{|c|}{$110^{0}$} \\
\hline No. of elements & 10 & 12 & 16 \\
\hline \multirow{16}{*}{ Excitation amplitudes $\left(a_{i}\right)$} & 0.6728 & 0.2699 & 0.5232 \\
\hline & 0.4838 & 0.4973 & 0.0718 \\
\hline & 0.2651 & 0.6247 & 0.3344 \\
\hline & 0.6880 & 0.6089 & 0.4895 \\
\hline & 0.2896 & 0.8114 & 0.6623 \\
\hline & 0.6536 & 0.2136 & 0.1877 \\
\hline & 0.5125 & 0.7713 & 0.8941 \\
\hline & 0.3148 & 0.8532 & 0.6679 \\
\hline & 0.4445 & 0.2267 & 0.5754 \\
\hline & 0.8132 & 0.8313 & 0.5494 \\
\hline & & 0.3379 & 0.6393 \\
\hline & & 0.8596 & 0.5239 \\
\hline & & & 0.3096 \\
\hline & & & 0.2282 \\
\hline & & & 0.2692 \\
\hline & & & 0.2932 \\
\hline
\end{tabular}

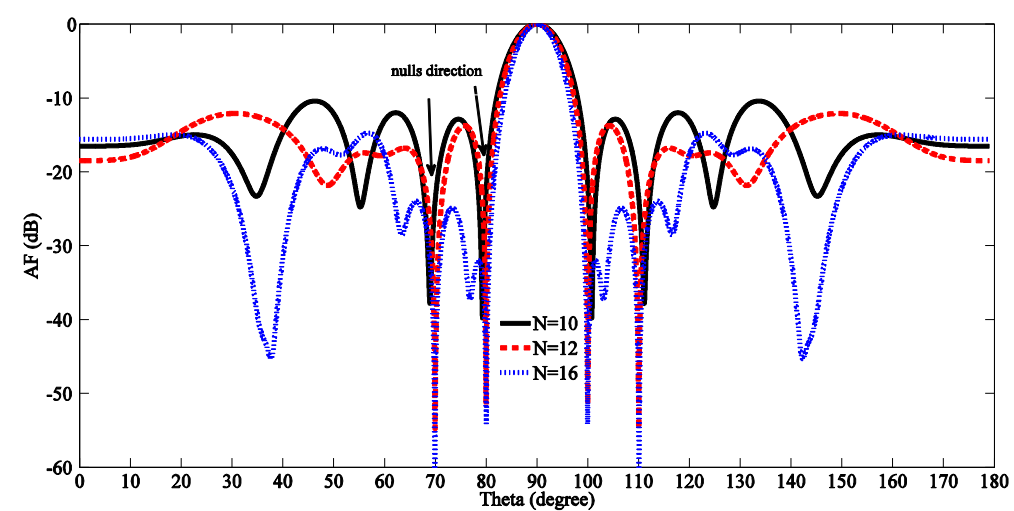

Fig. 11. The plot of AF when nulls are imposed at $70^{\circ}, 80^{\circ}, 100^{\circ}$ and at $110^{\circ}$.

TABLE X SLL, HPBW AND NULL DEPTH WHEN NULLS ARE IMPOSED AT $\theta=70^{\circ}, 80^{\circ}, 100^{\circ}$ AND $110^{\circ}$.

\begin{tabular}{|c|c|c|c|c|}
\hline $\mathrm{N}$ & HPBW (degree) & SLL $(\mathrm{dB})$ & Null depth at $70^{\circ}$ & Null depth at $80^{\circ}$ \\
\hline 10 & 9 & -10.4 & $-59.00 \mathrm{~dB}$ & $-32.1 \mathrm{~dB}$ \\
\hline 12 & 9 & -12 & $-51.55 \mathrm{~dB}$ & $-51.1 \mathrm{~dB}$ \\
\hline 16 & 7 & -16 & $-52 \mathrm{~dB}$ & $-53.3 \mathrm{~dB}$ \\
\hline
\end{tabular}

From the Table VII, \& X and from Fig. 10, \& 11, it has found that NULL can be placed in any angular direction by optimizing the excitation amplitude. The aim of nulls placement is used to reject the power where the interfered or unwanted signals are present. 


\section{CONCLUSION}

In this paper, it has developed the PSO algorithm code to calculate and optimize the inter-element spacing and excitation amplitude, which the optimization objectives targeted in this work are involved the HPBW, the SLL, directivity and null steering in certain angular directions. In order to achieve these goals, the two fitness functions have been used in the PSO method. One of the fitness functions is used with different values for the number of antenna array elements to find the optimum values of excitation amplitude and inter-element spacing that give a pattern having minimum SLL and HPBW. PSO employs the second fitness function to control the nulls in a specified direction and minimize SLL and HPBW by optimizing excitation amplitude.

\section{REFERENCES}

[1] B. Goswami and D. Mandal, "Nulls and Sidelobe Levels Control in a Time Modulated Linear Antenna Array by Optimizing Excitations and Element Locations Using RGA,” Journal of Microwaves, Optoelectronics, and Electromagnetic Applications, Vol. 12, No. 2, December 2013.

[2] T. Girard, R. Staraj, E. Cambiaggio and F. Muller, "A simulated annealing algorithm for planar or conformal antenna array synthesis with optimized polarization," Microwave and Optical Technology Letters, Vol. 28, No. 2, pp. 86-89, 2001.

[3] C. M. Coleman, E. J. Rothwell and J. E. Ross, "Investigation of simulated annealing, ant-colony optimization, and genetic algorithms for self-structuring antennas," IEEE Transactions on Antennas and Propagation, Vol. 52, No. 4, pp. 1007-1014, 2004.

[4] D. Marcano and F. Duran, "Synthesis of antenna arrays using genetic algorithms," IEEE Antennas and Propagation Magazine, Vol. 42, No. 3, pp. 12-20, 2000.

[5] D. I. Abu-Al-Nadi, T. H. Ismail and M. J. Mismar, "Synthesis of linear array and null steering with minimized side-lobe level using particle swarm optimization," Proceedings of the $4^{\text {th }}$ European Conference on Antennas and Propagation, pp. 1-4, 2010.

[6] M. M. Khodier and C. G. Christodoulou, "Linear array geometry synthesis with minimum sidelobe level and null control using particle swarm optimization," IEEE Transactions on Antennas and Propagation, Vol. 53, No. 8, pp. 2674-2679, 2005.

[7] J. Song, H. Zheng and L. Zhang, "Application of particle swarm optimization algorithm and genetic algorithms in beam broadening of phase array antenna," International Symposium on Signals, Systems and Electronics (ISSSE), pp. 17-20, 2010.

[8] M. Y. X. Lia, "Optimal synthesis of linear antenna array with composite differential evolutionary algorithm,” Scientia Iranica, Vol. 19, No. 9, pp. 1780-1787, 2012.

[9] B. Goswami and D. Mandal, "A genetic algorithm for the level control of nulls and side lobes in linear antenna arrays,” Journal of Computer and Information Sciences, Vol. 25, No. 2, pp. 117-126, 2013.

[10] S. K. Gotsis, K. Siakavara, E. Vafiadis and J. Sahalos, "A multi-objective approach to subarrayed linear antenna arrays design based on memetic differential evolution," IEEE Transactions on Antennas and Propagation, Vol. 61, pp. 3042-3052, 2013.

[11] A. Bhargav and N. Gupta, "Multiobjective genetic optimization of nonuniform linear Array with low sidelobes and beamwidth,” IEEE Antennas and Wireless Propagation Letters, Vol. 12, pp. 1547-1549, 2013.

[12] G. Ram, D. Mandal, R. Kar and S. P. Prasad, "Optimized hyper beamforming of receiving linear antenna arrays using Firefly algorithm,” International Journal of Microwave and Wireless Technologies, Vol. 6, pp. 181-194, 2014. 
[13] C. A. Balanis, “Antenna Theory Analysis and Design”, A John Wiley and sons, 2005.

[14] G. Ram, D. Mandal, R. Kar and S. P. Ghosal, "Synthesis of time modulated linear antenna arrays using particle swarm optimization,” IEEE Region 10 Conference TENCON, pp. 1-4, 2014.

[15] A. Banookh, and S. M. Barakati, "Optimal Design of Double Folded Stub Microstrip Filter by Neural Network Modelling and Particle Swarm Optimization," Journal of Microwaves, Optoelectronics and Electromagnetic Applications, Vol. 11, No. 1, June 2012.

[16] F. R. Durand, and T. Abrão, "Particle Swarm Optimization in WDM/OCDM Networks with Physical Impairments," Journal of Microwaves, Optoelectronics and Electromagnetic Applications, Vol. 12, No. 2, December 2013.

[17] C. J. A. Bastos-Filho and E. M. N. Figueiredo, "Design of Distributed Optical-Fiber Raman Amplifiers using Multi-objective Particle Swarm Optimization" Journal of Microwaves, Optoelectronics and Electromagnetic Applications, Vol. 10, No. 2, December 2011.

[18] A. Banookh, and S. M. Barakati, "Optimal Design of Double Folded Stub Microstrip Filter by Neural Network Modelling and Particle Swarm Optimization" Journal of Microwaves, Optoelectronics and Electromagnetic Applications, Vol. 11, No. 1, June 2012.

[19] L. Wakrim, S. Ibnyaich, and M. M. Hassani, "The study of the ground plane effect on a Multiband PIFA Antenna by using Genetic Algorithm and Particle Swarm Optimization” Journal of Microwaves, Optoelectronics and Electromagnetic Applications, Vol. 15, No. 4, December 2016.

[20] S. S, Travessa, and W. P. Carpes Jr., "Use of an Artificial Neural Network-based Metamodel to Reduce the Computational Cost in a Ray-tracing Prediction Model" Journal of Microwaves, Optoelectronics and Electromagnetic Applications, Vol. 15, No. 4, December 2016.

[21] P. J. Bevelacqua and C. A. Balanis, "Minimum side lobe level for linear array," IEEE Transactions on Antennas and Propagation, Vol. 55, No. 12, pp. 3442 - 3449, 2007.

[22] A. R. Harish and M. Sachidananda, Antennas and Wave Propagation, Oxford University Press, 2007.

[23] K. Kennedy and R. Eberhart, "Particle swarm optimization," Proceedings of the IEEE International Conference on Neural Networks, pp. 1942-1948, 1995.

[24] M. A. Haq, M. T. Afzal, U. Rafique, Q. D. Memon, M. A. Khan and M. M. Ahmed, "Log periodic dipole antenna design using particle swarm optimization," International Journal on Electromagnetics and Applications, Vol. 2, No. 4, pp. 65-68, 2012.

[25] Q. D. Memon, M. M. Ahmed, N. M. Memon and U. Rafique, "An efficient mechanism to simulate DC characteristics of GaAs MESFETs using swarm optimization," $9^{\text {th }}$ IEEE International Conference on Emerging Technologies (ICET), pp. 1-5, 2013.

[26] A.Sharaqa and N. Dib, "Design of linear and elliptical antenna arrays using Biogeography Based Optimization,” Arab Journal of Science and Engineering, Vol. No. 4,pp 2929-2939, 2013.

[27] G.Al-Kubati, "Central force optimization method and its application to the design of antennas," Master Thesis, Jordan University os science and Technology, 2009.

[28] P. Joshi, and N. Jain, "Optimization of linear antenna array using genetic algorithm for a reduction in side lobe levels and to improve directivity," International Journal of Latest Trends in Engineering and Technology (IJLTET), Vol. 2, No. 3, 2013. Vol. 28, pp 540-549, 2014.

[29] L. Pappula, and D. Ghosh, "Linear antenna array synthesis using Cat Swarm Optimization," International Journal of Electronics and Communication (AEU), Vol. 28, No. 6, pp 540-549, 2014.

[30] G. Ram, D. Mandal, R. Kar, S. P. Choshal, "Directivity maximization and optimal far-field pattern of time modulated linear antenna arrays using evolutionary algorithms," International Journal of Electronics and Communication (AEU), Vol. 69, pp 1800-1809, 2015. 